Rev. Int. Contam. Ambie. 35 (1) 65-79, 2019

DOI: 10.20937/RICA.2019.35.01.05

\title{
ADECUABILIDAD Y COMPARACIÓN DE TÉCNICAS ESPECTROSCÓPICAS PARA EL ANÁLISIS DE MUESTRAS DE ORIGEN GEOLÓGICO
}

\author{
Olivia ZAMORA-MARTÍNEZ ${ }^{1,2 *}$, Rufino LOZANO-SANTA CRUZ ${ }^{1,2}$, \\ Hugo Yuset SAMAYOA-OVIEDO ${ }^{1,2}$, Mariana VELÁZQUEZ-CASTRO ${ }^{1,2}$ y Blanca PRADO-PANO ${ }^{1,2}$
}

${ }^{1}$ Instituto de Geología, Universidad Nacional Autónoma de México, Ciudad Universitaria, 04510 Ciudad de México, México

${ }^{2}$ Laboratorio Nacional de Geoquímica y Mineralogía (LANGEM), Universidad Nacional Autónoma de México, Ciudad Universitaria, C.P. 04510, Ciudad de México, México

*Autora para correspondencia: oliviazm@geologia.unam.mx

(Recibido noviembre 2017; aceptado mayo 2018)

Palabras clave: espectroscopia analítica, adecuabilidad de métodos, rocas, suelos, sedimentos, lixiviados de mina

\section{RESUMEN}

Se optimizaron y adecuaron cuatro metodologías analíticas para la determinación de especies químicas de interés ambiental. Por medio de la técnica de espectroscopia de absorción atómica con flama (EAA-F) fue posible cuantificar de manera secuencial las concentraciones de $\mathrm{Fe}, \mathrm{Mn}, \mathrm{Cu}, \mathrm{Cr}, \mathrm{Ni}, \mathrm{Pb}$ y $\mathrm{Zn}$ en muestras de sedimentos, suelos y rocas. Mediante la formación de complejos coloridos de especies de hierro, obtenidos en condiciones de reacción específicas y bien definidas, y su posterior cuantificación mediante espectroscopia visible con metodologías independientes, fue posible la determinación del contenido de hierro total, $\mathrm{Fe}^{2+}$ y $\mathrm{Fe}^{3+}$ en lixiviados de mina. Las metodologías descritas resultaron ser precisas y exactas, se encuentran libres de interferencias de matriz y son comparables con otras metodologías ya validadas. Los intervalos de linealidad en EAA-F para los siete elementos varían de 0.05 a $16 \mathrm{mg} / \mathrm{L}$, con límites de detección comprendidos entre 0.03 y $0.2 \mathrm{mg} / \mathrm{L}$. Con relación a las determinaciones colorimétricas de $\mathrm{Fe}$ total, $\mathrm{Fe}^{2+}$ y $\mathrm{Fe}^{3+}$ en muestras de lixiviados mineros sulfurados, se observaron intervalos lineales de concentración comprendidos entre 0.05 y 17.5 $\mathrm{mg} / \mathrm{L}$. Los límites de detección obtenidos fueron de $0.05 \mathrm{mg} / \mathrm{L}$ para $\mathrm{Fe}$ total y $\mathrm{Fe}^{2+}$ y de $0.14 \mathrm{mg} / \mathrm{L}$ para $\mathrm{Fe}^{3+}$.

Key words: analytical spectroscopy, suitability of methods, rocks, soils, sediments, mine leached

\begin{abstract}
Four analytical methodologies for the determination of chemical species of environmental interest were optimized and adapted. By means of the flame atomic absorption spectroscopy technique (FAAS) it was possible to sequentially quantify $\mathrm{Fe}, \mathrm{Mn}, \mathrm{Cu}, \mathrm{Cr}$, $\mathrm{Ni}, \mathrm{Pb}$, and $\mathrm{Zn}$ concentrations in sediment, soil and rock samples. Through the formation of colorful complexes of iron species, obtained under specific and well-defined reaction conditions, and their subsequent quantification by visible spectroscopy with independent methodologies, it was possible to determine the total iron, $\mathrm{Fe}^{2+}$ and $\mathrm{Fe}^{3+}$ content in mine leachates. The methodologies described turned out to be precise and accurate, they are free of matrix interferences and are comparable with other validated methodologies. The linearity intervals in FAAS for the seven elements vary from 0.05
\end{abstract}


to $16 \mathrm{mg} / \mathrm{L}$, with detection limits between 0.03 and $0.2 \mathrm{mg} / \mathrm{L}$. With respect to the colorimetric determinations of total $\mathrm{Fe}, \mathrm{Fe}^{2+}$ and $\mathrm{Fe}^{3+}$ in samples of sulfided mining leachates, linear ranges of concentration comprised between 0.05 and $17.5 \mathrm{mg} / \mathrm{L}$ were observed. The detection limits obtained were $0.05 \mathrm{mg} / \mathrm{L}$ for total $\mathrm{Fe}$ and $\mathrm{Fe}^{2+}$ and $0.14 \mathrm{mg} / \mathrm{L}$ for $\mathrm{Fe}^{3+}$.

\section{INTRODUCCIÓN}

Para comprender los procesos ambientales de contaminación es fundamental analizar materiales de diferentes orígenes tales como agua, suelo, sedimentos, rocas, etc. Como parte de ello se requiere la determinación elemental de los componentes traza, menores y mayores de dichas matrices (Gómez-Nieto et al. 2015). El monitoreo de elementos potencialmente tóxicos (EPT) es fundamental (Balasubramanian y Pugalenthi 1999), ya que los metales pesados y el arsénico son de los principales contaminantes del medio ambiente (Sastre et al. 2002). Cabe mencionar que la industria metalúrgica genera diversos residuos entre los que destacan los jales mineros, cuya disposición final no adecuada puede tener un significativo impacto ambiental debido a los procesos de oxidación que lixivian sus componentes (Gray 1997, Cravotta y Trahan 1999, Jennings et al. 2000). Al ser los jales el producto de la trituración y molienda de las rocas y minerales de interés, una vez que se han extraído los metales económicos mediante procesos físicos y químicos, éstos tienen la misma composición química y mineralógica que el yacimiento de origen. Los estudios para controlar y estabilizar dichos residuos involucran una caracterización completa que contempla diversos parámetros como $\mathrm{pH}$, conductividad, sólidos totales disueltos (STD), potencial de óxido-reducción (Eh), composición elemental total y especiación química, entre otros (Zamora 2003).

Dentro de las técnicas analíticas más utilizadas para la elucidación de la composición química de una muestra de origen ambiental, se pueden mencionar a la espectrometría de absorción atómica, la fluorescencia de rayos X, la espectroscopia visible y la espectrometría de emisión atómica con plasma acoplado inductivamente. Debido a que en muchas ocasiones es posible utilizar más de uno de estos procesos para realizar la misma determinación química, es de primordial importancia seleccionar la mejor opción de análisis. Por ello es necesario tomar en cuenta, entre otros elementos, el origen de la muestra, su preparación, el nivel de concentración esperado del analito, la presencia de interferencias de matriz, la cantidad de la muestra con la que se cuenta y la productividad de la técnica de análisis, que en conjunto son puntos clave para la obtención de resultados confiables. Este aspecto de la práctica de la química analítica se ha vuelto cada vez más importante, ya que los organismos oficiales, con la finalidad de establecer políticas o vigilar su cumplimiento, han implantado lineamientos más complejos y exigentes para asegurar que las mediciones analíticas sean cada vez más confiables. Para lograr lo anterior es necesario demostrar que los procedimientos analíticos son aptos para el uso indicado, lo que es conocido como validación del método analítico (Christian 2009).

La espectrometría de absorción atómica (EAA) es utilizada como técnica de análisis elemental para matrices de tipo ambiental (Chen y Teo 2001). Permite analizar hasta 70 elementos (metales y no metales) en prácticamente todo tipo de muestras (ambientales, biológicas, farmacéuticas, etc.) con límites de detección hasta de microgramos/litro cuando se utiliza el horno de grafito como fuente de atomización de la muestra. Sin embargo, debido a que las concentraciones de los EPT en minerales y residuos pueden ser de varios cientos de partes por millón (Singh y Agrawal 2012, Gao et al. 2013), es común utilizar la flama como fuente de atomización (Skoog et al. 2001). La EAA presenta intervalos lineales de concentración restringidos y sólo es posible la determinación monoelemental secuencial y no, como en otras técnicas, la determinación simultánea, lo cual dificulta su universalidad (Harris 2013, Gómez-Nieto et al. 2015). Para realizar el análisis de una muestra es necesario que ésta se encuentre en forma líquida y libre de sólidos, por lo que es inevitable una preparación que involucre desde la digestión ácida de la muestra, asistida por microondas, hasta el paso de filtración a través de una membrana, con tiempos de preparación relativamente cortos $(1 \mathrm{~h})$. Los programas de digestión se realizan sin supervisión constante y presentan porcentajes de recobro satisfactorios (Skoog et al. 2001, Soylak et al. 2007, Harris 2013, Soodan et al. 2014). En un intento de armonizar los protocolos de digestión, la Agencia de Protección Ambiental de Estados Unidos (USEPA, por sus siglas en inglés) ha recomendado el uso de ácido nítrico para la digestión parcial de matrices sólidas como 
suelos, lodos y sedimentos, con lo cual es posible solubilizar elementos que no se encuentran enlazados dentro de la estructura de los silicatos contenida en dichas matrices (USEPA 1996a). Cabe mencionar que el uso de ácido fluorhídrico (HF) proporciona un esquema con el cual la digestión es total (Sastre et al. 2002), pero su uso requiere de recipientes para microondas de composición polimérica inerte y su análisis, de un sistema de introducción de la muestra compatible (USEPA 1996b).

La fluorescencia de rayos X (FRX) es una técnica igualmente difundida para el análisis cuantitativo de materiales de origen geológico. Requiere de poca manipulación de la muestra y tiempos de análisis relativamente cortos. La generación de los resultados finales se apoya en muchos casos en el uso de programas de lectura corregidos, que eliminan o minimizan las interferencias elementales (Brouwer 2013).

La espectroscopia visible es una técnica de medición que proporciona información acerca de la composición y la concentración de especies químicas coloridas que absorben la radiación electromagnética comprendida entre 400 y $1100 \mathrm{~nm}$. La determinación suele ser rápida aun cuando sea necesario hacer reaccionar el analito de interés para producir una especie que absorba en el visible. Se trata de una técnica que también puede presentar interferencias (CENAM-EMA 2008).

La espectroscopia de emisión óptica con plasma acoplado inductivamente (ICP-OES, por sus siglas en inglés) es una de las técnicas más utilizadas para la determinación de trazas de elementos en una gran variedad de matrices, las cuales comúnmente deben digerirse previamente igual que en la EAA. La ICPOES se basa en la emisión espontánea de fotones de los átomos que han sido excitados por un plasma de argón de alta temperatura $(6800 \mathrm{~K})$, cuya señal es característica de cada elemento y en ciertas condiciones proporcional a la concentración. Los equipos analíticos actuales permiten la medición simultánea de más de 70 elementos, aunque los parámetros de desempeño de la técnica deben evaluarse para cada una de las matrices de trabajo, ya que existen interferencias espectrales que deben corregirse (Jimeno 2017).

Con el objetivo de obtener mediciones analíticas consistentes, confiables y exactas, se requiere de métodos analíticos validados (Ata et al. 2015). Los parámetros de desempeño dependen del tipo de análisis que se realice (Magnusson and Örnemark 2014, CCAyA 2011), por lo que siempre será necesario realizar la optimización de un método de cuantificación para un fin específico y determinar su adecuabilidad.
De acuerdo con lo anterior, en este trabajo se compara el desempeño de cuatro metodologías analíticas para el análisis de muestras de origen geológico, las cuales fueron evaluadas, adecuadas y optimizadas en matrices de trabajo específico.

\section{MATERIALES Y MÉTODOS}

\section{Descripción de las muestras de origen geológico de trabajo}

Como muestras de trabajo para los análisis de EAA-F y FRX con dispersión de longitud de onda (WD, por sus siglas en inglés) se utilizaron $10 \mathrm{ma}-$ teriales de referencia geológicos sólidos: IGLa-1, IGLs-1, SdAR-1, SdAR-L2, SdAR-M2, GS-N, IGLsy-1, IGLsy-2, IGLsy-4 e IGLgb-3. Éstos comprenden suelos, sedimentos y rocas descritos en Potts et. al. (1992), Lozano y Bernal (2005) y en los reportes de ejercicios interlaboratorios del programa GeoPT coordinados por la Open University de Londres y la Asociación Internacional de Geoanalistas (IAG).

Para los análisis de especiación de hierro por medio de espectroscopia visible y la cuantificación del contenido de Fe total por ICP-OES, se utilizaron 57 muestras de lixiviados, las cuales fueron producidas mediante el contacto del drenaje ácido de mina proveniente de jales mineros sulfurados y minerales alcalinos (eutita, filita y piedra caliza), procedentes de la zona norte de México. Estas muestras fueron acidificadas y conservadas en refrigeración a $4{ }^{\circ} \mathrm{C}$ hasta su análisis.

\section{Técnicas analíticas \\ Espectroscopia de absorción atómica con flama (EAA-F)}

Por medio de esta técnica se analizaron los siguientes elementos químicos: $\mathrm{Fe}, \mathrm{Mn}, \mathrm{Cu}, \mathrm{Cr}, \mathrm{Ni}$, $\mathrm{Pb}$ y $\mathrm{Zn}$. Se prepararon disoluciones patrón de cada elemento mediante la dilución de un estándar de $1000 \mathrm{mg} / \mathrm{L}$ en $\mathrm{HNO}_{3}$ al $2 \%$ (Sigma-Aldrich, grado ACS) en agua ultrapura de resistividad no menor a 18.2 $\mathrm{M} \Omega \mathrm{cm}$ (Milli-Q). Para $\mathrm{Cu}, \mathrm{Cr}, \mathrm{Ni}, \mathrm{Pb}$ y Fe se contó con disoluciones de materiales de referencia certificados de dicha concentración (PerkinElmer), mientras que los estándares de Mn y $\mathrm{Zn}$ fueron preparados con una sal grado reactivo analítico del elemento $\left(\mathrm{MnCl}_{2} \cdot 4 \mathrm{H}_{2} \mathrm{O}>99.6 \%\right.$ y $\mathrm{ZnSO}_{4}>99.0 \%$, ambas Merck). Las determinaciones se realizaron con un equipo de absorción atómica marca Perkin Elmer modelo $900 \mathrm{H}$ en modalidad flama con lámparas de cátodo hueco y con corrección de fondo de deuterio; la medición se hizo en la respectiva 
línea de resonancia y con una flama compuesta de aire-acetileno. Para cada elemento se optimizaron y adecuaron las condiciones para su cuantificación (Cuadro I).

\section{Determinación de parámetros de desempeño}

Una vez realizada la optimización de la metodología, se determinaron los intervalos lineales de concentración para cada uno de los analitos. Los parámetros utilizados para la evaluación de la linealidad fueron: 1) un coeficiente de correlación (r) mayor o igual a 0.99 , y 2) el cumplimiento de la aleatoriedad de los residuos en las pruebas no paramétricas de rachas de cada una de las curvas obtenidas (CCAyAC 2011, Miller y Miller 2002, Walpole et al. 2012).

Se determinó la precisión para tres niveles de concentración de cada analito, considerando un valor de baja concentración, uno intermedio y uno alto, todos ellos dentro del intervalo lineal de cada elemento (CCAyAC 2011). Cada concentración fue preparada por quintuplicado de manera independiente. La evaluación de este parámetro se realizó mediante el cálculo del porcentaje de desviación estándar relativa (\% DER) de la señal analítica obtenida (absorbancia):

$\% \operatorname{Der}=\frac{s}{\bar{x}} \times 100$

donde $s$ es la desviación estándar de las mediciones y $\bar{x}$ es el promedio de éstas.

El parámetro de exactitud también fue calculado para tres niveles de concentración, un valor bajo, uno intermedio y uno alto, todos ellos dentro del intervalo lineal de cada elemento (CCAyAC 2011). En este caso los valores de la señal analítica obtenidos fueron convertidos en unidades de concentración mediante el uso de los parámetros de regresión calculados de las respectivas curvas de calibración, para finalmente ser comparados con el valor conocido (Magnusson and Örnemark 2014).
Respecto a los límites de detección (LD) y de cuantificación (LC), éstos fueron determinados utilizando la señal analítica que se obtiene al analizar disoluciones de una muy baja concentración del elemento de trabajo. Para su obtención se realizaron siete inyecciones de disoluciones de concentración conocida de cada uno de los analitos $(0.02 \mathrm{mg} / \mathrm{L}$ para $\mathrm{Mn}$ y Cu, $0.04 \mathrm{mg} / \mathrm{L}$ para Fe, $0.05 \mathrm{mg} / \mathrm{L}$ para Ni y Zn y $0.20 \mathrm{mg} / \mathrm{L}$ para Cr y Pb). Con los valores de absorbancia determinados, se calcularon la media $(\bar{x})$ y la desviación estándar $(s)$ de cada elemento. El límite de detección se reporta de acuerdo con la ecuación:

$L D=\bar{x}+3 s$

En tanto que el límite de cuantificación se reporta como:

$L C=\bar{x}+10 s$

donde $(\bar{x})$ es el promedio de las mediciones y $s$ la desviación estándar de éstas.

Posteriormente se obtuvieron los valores de los límites de detección y de cuantificación en unidades de concentración con la ecuación de la respectiva curva de calibración (Miller y Miller 2002).

\section{Evaluación de interferencias}

Con el objeto de evaluar la presencia de interferencias de matriz durante la cuantificación de los siete elementos de estudio mediante EAA-F se fortificaron por triplicado, en tres niveles de concentración, los digeridos provenientes del tratamiento por digestión ácida asistida por microondas de materiales de referencia geológicos. Se eligió el material de referencia GS-N para la determinación de Fe, mientras que el IGLgb-3 sirvió para las lecturas de Zn y Mn, y el SdAR-1 se utilizó para el resto de los analitos en estudio. Las concentraciones de

CUADRO I. PARÁMETROS DE TRABAJO PARA LA DETERMINACIÓN DE Cr, Cu, Pb, Zn, Mn, Ni y Fe POR MEDIO DE ESPECTROSCOPIA DE ABSORCIÓN ATÓMICA CON FLAMA

\begin{tabular}{lccccc}
\hline Analito & $\begin{array}{c}\text { Longitud de onda } \\
(\mathrm{nm})\end{array}$ & $\begin{array}{c}\text { Intensidad de corriente } \\
\text { en la lámpara }(\mathrm{mA})\end{array}$ & $\begin{array}{c}\text { Rendija } \\
(\mathrm{nm})\end{array}$ & & \multicolumn{2}{c}{ Flujo de los gases (L/min) } \\
\cline { 5 - 6 } & & 10 & 0.7 & Aire & Acetileno \\
\hline $\mathrm{Cr}$ & 357.87 & 9 & 0.7 & 10.0 & 4.0 \\
$\mathrm{Cu}$ & 324.75 & 10 & 0.7 & 10.0 & 2.5 \\
$\mathrm{~Pb}$ & 283.31 & 15 & 0.7 & 10.0 & 2.5 \\
$\mathrm{Zn}$ & 213.86 & 20 & 0.2 & 10.0 & 2.5 \\
$\mathrm{Mn}$ & 279.48 & 25 & 0.2 & 10.0 & 2.5 \\
$\mathrm{Ni}$ & 232.00 & 25 & 0.2 & 10.0 & 2.5 \\
$\mathrm{Fe}$ & 248.33 & & & &
\end{tabular}


fortificación utilizadas se encuentran comprendidas en el intervalo de 0.13 a $15.15 \mathrm{mg} / \mathrm{L}$ y éstas fueron seleccionadas respecto al intervalo lineal de cada elemento.

Tanto los digeridos sin fortificar como los fortificados fueron analizados como muestras por medio de EAA-F. Su concentración fue determinada con el uso de su respectiva curva de calibración. La evaluación de interferencias de matriz se presenta como la recuperación obtenida para cada analito en los tres niveles de concentración de trabajo y se calcula de acuerdo con la ecuación 4 (CCAyAC 2011).

$\%$ Recuperación $=\frac{(\text { Cmuestra fortificada }- \text { Cmuestra })}{\text { Cadicionada }} \times 100$

donde Cmuestra fortificada es la concentración determinada en el digerido fortificado; Cmuestra es la concentración del digerido sin fortificar, y Cadicionada es el nivel de concentración utilizada para fortificar

\section{Intercomparación de metodologías}

Finalmente se realizó una comparación entre dos técnicas de análisis para la cuantificación de los contenidos de $\mathrm{Fe}, \mathrm{Mn}, \mathrm{Cu}, \mathrm{Cr}, \mathrm{Ni}, \mathrm{Pb}$ y $\mathrm{Zn}$ presentes en los 10 materiales de referencia geológicos sólidos descritos con anterioridad. La comparación involucra la metodología de EAA-F descrita en el presente trabajo y FRX-WD, una segunda técnica de análisis ya confirmada en otro laboratorio.

Para realizar los análisis de EAA-F fue necesario someter cada uno de los materiales de referencia a un proceso de digestión para solubilizar sus componentes. Dicho procedimiento consistió en adicionar $10 \mathrm{~mL}$ de $\mathrm{HNO}_{3}$ concentrado a $0.5 \mathrm{~g}$ del material sólido, dejar reposar la mezcla durante $2 \mathrm{~h}$ y posteriormente someterla a una digestión en microondas utilizando el equipo Multiwave 3000 de Anton Paar con rotor 48MF50. El programa de digestión utilizado fue el indicado en el método USEPA 3050B (USEPA1996a), que consiste en aplicar una rampa de temperatura hasta un máximo de $180^{\circ} \mathrm{C}$ en 5.5 min, un tiempo de mantenimiento de $9.5 \mathrm{~min} y$ finalmente un decremento de la potencia a $0 \mathrm{~W}$ (enfriamiento) durante $20 \mathrm{~min}$. El digerido obtenido fue percolado a través de papel filtro cuantitativo Whatman número 41, con tamaño de poro de 20 a $25 \mu \mathrm{m}$, libre de cenizas. El filtrado fue transferido cuantitativamente a un matraz volumétrico de 50 $\mathrm{mL}$, llevando la mezcla a la marca del aforo con agua desionizada. Cada uno de los materiales de referencia se digirió por triplicado. Para la determinación de las concentraciones de los siete analitos en las muestras digeridas se realizaron, cuando fue necesario, las diluciones pertinentes para que la señal obtenida pudiera interpolarse en la respectiva curva de calibración del elemento de estudio. Posteriormente, las concentraciones obtenidas en fase líquida $(\mathrm{mg} / \mathrm{L})$ fueron convertidas a $\mathrm{mg} / \mathrm{kg}$ empleando la masa del material utilizado para la digestión.

La preparación de los materiales para realizar los análisis de FRX-WD consistió en pesar $5.6 \mathrm{~g}$ del material sólido y mezclarlos con $1.0 \mathrm{~g}$ de aglutinante para FRX (cera-C micropolvo) en un mortero de ágata hasta obtener una mezcla homogénea en forma de polvo fino. Posteriormente, la mezcla se comprimió a 20 ton $/ \mathrm{cm}^{2}$ durante $45 \mathrm{~s}$ para obtener una tableta de $3 \mathrm{~cm}$ de diámetro (Lozano y Bernal 2005).

El análisis de los materiales de trabajo se llevó a cabo mediante el uso de curvas de calibración monoelementales para FRX-WD. Éstas se generaron mediante el uso de 19 materiales de referencia certificados: AGV-1, Es-5, Es-15, JA-2, JG-2, JR-3, MICA-Mg, RGM-1, SDC-1, SDO-1, SIEM-09, QLO-1, SCo-1, SGR-1b, W-2a, BX-N, JSd-2, FK-N y DTS-1 de acuerdo con lo reportado por Lozano y Bernal (2005). Se utilizó un espectrómetro secuencial de fluorescencia de rayos X marca Rigaku, modelo Primus II, equipado con un tubo de rodio y una ventana de berilio de $30 \mu \mathrm{m}$. En el cuadro II se resumen las condiciones de trabajo utilizadas para cada una de las determinaciones.

\section{Espectroscopia visible}

Por medio de esta técnica se determinaron los contenidos de $\mathrm{Fe}$ total, $\mathrm{Fe}^{2+}$ y $\mathrm{Fe}^{3+}$ mediante la formación de complejos coloridos que absorben en la región del visible. Se prepararon disoluciones patrón para cada analito mediante la dilución de un estándar que contenía aproximadamente 1000 $\mathrm{mg} / \mathrm{L}$ en agua ultrapura de resistividad no menor a $18.2 \mathrm{M} \Omega \mathrm{cm}$ (Milli-Q). Estos estándares fueron preparados a partir de sales grado reactivo analítico $\left(\mathrm{FeSO}_{4} \cdot 7 \mathrm{H}_{2} \mathrm{O}>99 \%\right.$ para Fe ${ }^{2+}$ y $\mathrm{Fe}\left(\mathrm{NO}_{3}\right)_{3} \cdot 9 \mathrm{H}_{2} \mathrm{O}>$ $98 \%$ para $\mathrm{Fe}^{3+}$, ambas de la marca Sigma Aldrich). Las determinaciones se realizaron con un espectrofotómetro UV-Visible, marca Thermo Scientific, modelo Evolution 201.

\section{Optimización de las condiciones de trabajo}

La cuantificación del contenido total de hierro se llevó a cabo en un medio reductor establecido por la presencia de hidroxilamina (a partir de $\mathrm{NH}_{2} \mathrm{OH} \cdot \mathrm{HCl}$ $>99.1 \%$, Sigma Aldrich) y la posterior adición de ortofenantrolina (oFen $\geq 99 \%$, Sigma Aldrich), reacciones 1 y 2 (Harvey et al. 1955). 
CUADRO II. PARÁMETROS DE TRABAJO PARA LA DETERMINACIÓN DE Cr, Cu, Pb, Zn, Mn, Ni y Fe POR MEDIO DE FLUORESCENCIA DE RAYOS X CON DISPERSIÓN DE LONGITUD DE ONDA

\begin{tabular}{|c|c|c|c|c|c|}
\hline Analito & Fuente de excitación & $\begin{array}{l}\text { Potencia de } \\
\text { excitación }\end{array}$ & $\begin{array}{l}\text { Cristal } \\
\text { analizador }\end{array}$ & $\begin{array}{l}\text { Línea } \\
\text { espectral }\end{array}$ & $\begin{array}{l}\text { Corrección } \\
\text { espectral }\end{array}$ \\
\hline $\mathrm{Fe}$ & $\begin{array}{l}\text { Tubo de rodio con ventana } \\
\text { de } 30 \mu \mathrm{m}\end{array}$ & $50 \mathrm{kV}-60 \mathrm{~mA}$ & LiF 200 & $\mathrm{Fe}-\mathrm{K} \alpha$ & $\begin{array}{c}\mathrm{Cr} \\
\mathrm{Ni} \\
\mathrm{Cu} \\
\text { Aglutinante (cera } \\
\mathrm{C} \text { micropolvo) }\end{array}$ \\
\hline $\mathrm{Ni}$ & $\begin{array}{l}\text { Tubo de rodio con ventana } \\
\text { de } 30 \mu \mathrm{m}\end{array}$ & $50 \mathrm{kV}-60 \mathrm{~mA}$ & LiF 200 & $\mathrm{Ni}-\mathrm{K} \alpha$ & $\begin{array}{l}\mathrm{Fe} \\
\mathrm{Cr}\end{array}$ \\
\hline $\mathrm{Cu}$ & $\begin{array}{l}\text { Tubo de rodio con ventana } \\
\text { de } 30 \mu \mathrm{m}\end{array}$ & $50 \mathrm{kV}-60 \mathrm{~mA}$ & LiF 200 & $\mathrm{Cu}-\mathrm{K} \alpha$ & $\begin{array}{l}\mathrm{Fe} \\
\mathrm{Ni}\end{array}$ \\
\hline $\mathrm{Zn}$ & $\begin{array}{l}\text { Tubo de rodio con ventana } \\
\text { de } 30 \mu \mathrm{m}\end{array}$ & $50 \mathrm{kV}-60 \mathrm{~mA}$ & LiF 200 & $\mathrm{Zn}-\mathrm{K} \alpha$ & $\begin{array}{l}\mathrm{Fe} \\
\mathrm{Cu}\end{array}$ \\
\hline $\mathrm{Pb}$ & $\begin{array}{l}\text { Tubo de rodio con ventana } \\
\text { de } 30 \mu \mathrm{m}\end{array}$ & $50 \mathrm{kV}-60 \mathrm{~mA}$ & LiF 200 & $\mathrm{~Pb}-\mathrm{L} \beta 1$ & $\begin{array}{c}\mathrm{Fe} \\
\mathrm{Mn} \\
\mathrm{Zn}\end{array}$ \\
\hline $\mathrm{Mn}$ & $\begin{array}{l}\text { Tubo de rodio con ventana } \\
\text { de } 30 \mu \mathrm{m}\end{array}$ & $50 \mathrm{kV}-60 \mathrm{~mA}$ & LiF 200 & $\mathrm{Mn}-\mathrm{K} \alpha$ & Sin corrección \\
\hline
\end{tabular}

La determinación de $\mathrm{Fe}^{2+}$ se realizó con la adición directa de oFen, reacción 2 (Harvey et al. 1955), mientras que la cuantificación de $\mathrm{Fe}^{3+}$ se logró mediante la formación de un complejo con tiocianato (a partir de $\mathrm{NaSCN} \geq 98 \%$, Sigma Aldrich), reacción 3 , de acuerdo con lo reportado por Charlot (1964) y Walton y Reyes (1983).

$2 \mathrm{NH}_{2} \mathrm{OH}+4 \mathrm{Fe}^{3+} \rightleftarrows 4 \mathrm{Fe}^{2+} \mathrm{N}_{2} \mathrm{O}+\mathrm{H}_{2} \mathrm{O}+4 \mathrm{H}^{+}$

$\mathrm{Fe}^{2+}+3$ oFen $\rightleftarrows\left[\mathrm{Fe}(\mathrm{oFen})_{3}\right]^{2+}$

$\mathrm{Fe}^{3+}+6 \mathrm{SCN}^{-} \rightleftarrows\left[\mathrm{Fe}(\mathrm{SCN})_{6}\right]^{3-}$

Con la finalidad de encontrar los máximos de absorción de cada complejo, a una disolución que contenía el equivalente a $10 \mathrm{mg} / \mathrm{L}$ de hierro complejado, se le realizó un barrido de longitud de onda comprendido entre 400 y $750 \mathrm{~nm}$.

Para la cuantificación de $\mathrm{Fe}^{2+}$ se evaluaron tres concentraciones de o-fenantrolina: $4.4 \times 10^{-4}, 8$ $\times 10^{-4}$ y $2.5 \times 10^{-3} \mathrm{M}$ a un valor de $\mathrm{pH}$ de 9.0 , el cual es fijado por el medio. Con cada concentración se prepararon curvas de calibración para el intervalo comprendido entre 0.01 y $20 \mathrm{mg} / \mathrm{L}$ de Fe. La concentración de oFen que presentó el valor de pendiente más alto fue seleccionada, y con ella se evaluó posteriormente la formación del complejo a $\mathrm{pH} 4.2$, que corresponde al valor de $\mathrm{pH}$ reportado en la literatura y que es fijado con una disolución amortiguadora de acetatos de concentración $0.1 \mathrm{M}$ (Arango et al. 2012).

Respecto al análisis de Fe total se seleccionó la concentración de oFen equivalente a $2.5 \times 10^{-3} \mathrm{M}$ y se evaluaron dos concentraciones de $\mathrm{NH}_{2} \mathrm{OH}$ : 1 y $2 \% \mathrm{~m} / \mathrm{v}$. Con éstas se prepararon curvas de calibración para el intervalo comprendido entre $0.01 \mathrm{y}$ $20 \mathrm{mg} / \mathrm{L}$ de $\mathrm{Fe}$, al valor de $\mathrm{pH}$ fijado por el medio (8.4). Se seleccionó la concentración de $\mathrm{NH}_{2} \mathrm{OH}$ que presentara mayor sensibilidad y con ella se verificó posteriormente la formación del complejo a $\mathrm{pH} 4.2$, de acuerdo con lo reportado por Arango et al. (2012).

Como primera parte de la cuantificación de $\mathrm{Fe}^{3+}$ se realizó la evaluación de tres concentraciones de tiocianato $\left(\mathrm{SCN}^{-}\right): 0.05,0.20$ y $0.45 \mathrm{M}$, al pH impuesto por el medio (4.0). Con el valor de concentración de $\mathrm{SCN}^{-}$que presentó la mejor sensibilidad, se verificó la influencia del pH sobre la formación del complejo, ajustando la mezcla de la reacción a $\mathrm{pH}<1$ con ácido nítrico $2 \mathrm{M}$ de acuerdo con lo reportado por Wood y Mellon (1941). Las condiciones optimizadas para la cuantificación de $\mathrm{Fe}$ total, $\mathrm{Fe}^{2+}$ y $\mathrm{Fe}^{3+}$ se resumen en el cuadro III. 
CUADRO III. PARÁMETROS DE TRABAJO OPTIMIZADOS PARA LA DETERMINACIÓN DE LOS CONTENIDOS DE Fe TOTAL, $\mathrm{Fe}^{2+} \mathrm{Y} \mathrm{Fe}^{3+}$ POR MEDIO DE ESPECTROSCOPIA VISIBLE

\begin{tabular}{llcc}
\hline Analito & Metodología & $\begin{array}{c}\text { Longitud de } \\
\text { onda }(\mathrm{nm})\end{array}$ & $\mathrm{pH}$ \\
\hline Fe total & oFen $\left(2.5 \times 10^{-3} \mathrm{M}\right)+\mathrm{NH}_{2} \mathrm{OH}(2 \%)$ & 511 & 4.2 y 8.4 \\
$\mathrm{Fe}^{2+}$ & oFen $^{-}\left(2.5 \times 10^{-3} \mathrm{M}\right)$ & 511 & 4.2 y 9.0 \\
$\mathrm{Fe}^{3+}$ & $\mathrm{SCN}^{-}(0.45 \mathrm{M})+\mathrm{HNO}_{3}(2 \mathrm{M})$ & 473 & $<1$ \\
\hline
\end{tabular}

Determinación de parámetros de desempeño

Una vez optimizadas las condiciones de trabajo para cada análisis, se determinaron el intervalo lineal, la precisión, la exactitud y los límites de detección y de cuantificación para cada analito, utilizando los criterios descritos para los análisis de EAA-F.

Para el cálculo del LD y del LC se utilizaron disoluciones de muy baja concentración del analito, que generaran una señal analítica diferenciable del ruido: $0.01 \mathrm{mg} / \mathrm{L}$ para Fe total, $0.02 \mathrm{mg} / \mathrm{L}$ para $\mathrm{Fe}^{2+}$ y $0.05 \mathrm{mg} / \mathrm{L}$ para $\mathrm{Fe}^{3+}$.

\section{Evaluación de interferencias}

Se preparó una mezcla de los lixiviados de mina referidos anteriormente, a la que se le añadieron concentraciones conocidas de $\mathrm{Fe}$ total, $\mathrm{Fe}^{2+}$ y $\mathrm{Fe}^{3+}$, de manera independiente y en 3 niveles de concentración. Se procedió a la determinación de los tres analitos en las muestras antes y después de fortificar, utilizando las metodologías descritas previamente.

Las concentraciones de hierro utilizadas para la fortificación fueron: $0.5,7.0$ y $17.5 \mathrm{mg} / \mathrm{L}$ para Fe total y $\mathrm{Fe}^{2+}$, y $0.4,7.0$ y $13.0 \mathrm{mg} / \mathrm{L}$ para Fe ${ }^{3+}$. Cada nivel de fortificación se preparó por triplicado. Su concentración fue determinada con el uso de la respectiva curva de calibración. La evaluación de las interferencias de matriz se presenta como la recuperación obtenida para cada analito en los tres niveles de concentración de trabajo y se calcula de acuerdo con la ecuación 4.

\section{Intercomparación de metodologías}

Los resultados obtenidos de la concentración de Fe total en las 57 muestras provenientes de lixiviados de mina, usando la metodología de espectroscopia visible adaptada en el presente trabajo, se compararon con los obtenidos por ICP-OES como técnica confirmatoria y previamente validada por otro laboratorio.

Para determinar la concentración de Fe por ICP-OES se realizó la introducción directa de los lixiviados. Las señales obtenidas se interpolaron en la curva de calibración generada con estándares de hierro, preparados mediante la dilución en $\mathrm{HNO}_{3}$ al
$2 \%$ (Sigma-Aldrich, grado ACS) en agua ultrapura de resistividad no menor a $18.2 \mathrm{M} \Omega \mathrm{cm}$ (Milli-Q), de un material de referencia de Fe $(1000 \mathrm{mg} / \mathrm{L}$, Perkin Elmer).

El equipo utilizado fue un espectrómetro de emisión atómica con plasma acoplado inductivamente marca Perkin Elmer, modelo OPTIMA 4300 DV. Las condiciones instrumentales se resumen en el cuadro IV.

CUADRO IV. PARÁMETROS DE TRABAJO PARA LA DETERMINACIÓN DE Fe TOTAL POR MEDIO DE ESPECTROSCOPIA DE EMISIÓN ÓPTICA CON PLASMA ACOPLADO INDUCTIVAMENTE

\begin{tabular}{|c|c|}
\hline Parámetro & Descripción \\
\hline Antorcha & cuarzo \\
\hline Flujo de Ar & $15 \mathrm{~L} / \mathrm{min}$ \\
\hline Visión de lectura & axial o radial \\
\hline Detector & $\begin{array}{l}\text { transferencia de carga } \\
\text { segmentada. }\end{array}$ \\
\hline Flujo del nebulizador & $0.65 \mathrm{~L} / \mathrm{min}$ \\
\hline Flujo gas auxiliar & $0.20 \mathrm{~L} / \mathrm{min}$ \\
\hline Potencia de RF & $1300 \mathrm{~W}$ \\
\hline Nebulizador & Gem Cone \\
\hline Velocidad bomba peristáltica & $1.8 \mathrm{~mL} / \mathrm{min}$ \\
\hline Longitud de onda de emisión de Fe & $238.204 \mathrm{~nm}$ \\
\hline Modo de lectura & Axial \\
\hline
\end{tabular}

RF: radiofrecuencia

\section{RESULTADOS Y DISCUSIÓN}

\section{Espectroscopia de absorción atómica con flama (EAA-F) \\ Determinación de los parámetros de desempeño}

En el cuadro $\mathbf{V}$ se resumen los parámetros de linealidad obtenidos para cada elemento. Se observa que la amplitud del intervalo de concentraciones depende de la sensibilidad del analito y se percibe que abarca aproximadamente dos órdenes de magnitud. 
CUADRO V. ANÁLISIS DE REGRESIÓN LINEAL PARA LOS SIETE ANALITOS AL $95 \%$ DE CONFIANZA, PARA 10 NIVELES DE CONCENTRACIÓN $(\mathrm{n}=3)$

\begin{tabular}{lcrcc}
\hline \multirow{2}{*}{ Analito } & \multicolumn{3}{c}{ Parámetro } \\
\cline { 2 - 5 } & Intervalo lineal $(\mathrm{mg} / \mathrm{L})$ & Ordenada al origen & Pendiente $(\mathrm{L} / \mathrm{mg})$ & Coeficiente de correlación $(\mathrm{r})$ \\
$\mathrm{Cr}$ & $0.28-4.00$ & $0.0082 \pm 0.0036$ & $0.0440 \pm 0.0017$ & 0.9987 \\
$\mathrm{Cu}$ & $0.28-4.00$ & $-0.0035 \pm 0.0046$ & $0.1340 \pm 0.0022$ & 0.9997 \\
$\mathrm{~Pb}$ & $0.70-16.00$ & $0.0012 \pm 0.0016$ & $0.0160 \pm 0.0002$ & 0.9999 \\
$\mathrm{Zn}$ & $0.075-1.00$ & $0.0078 \pm 0.0025$ & $0.2120 \pm 0.0048$ & 0.9992 \\
$\mathrm{Fe}$ & $0.12-4.00$ & $-0.0010 \pm 0.0021$ & $0.0750 \pm 0.0012$ & 0.9996 \\
$\mathrm{Mn}$ & $0.06-3.00$ & $0.0007 \pm 0.0035$ & $0.1750 \pm 0.0026$ & 0.9997 \\
$\mathrm{Ni}$ & $0.05-3.50$ & $0.0044 \pm 0.0016$ & $0.0654 \pm 0.0010$ & 0.9997 \\
\hline
\end{tabular}

Se puede observar que el analito más sensible para su determinación es el Zn, ya que posee la mayor pendiente en comparación con los otros elementos determinados, en tanto que el $\mathrm{Pb}$ es el elemento menos sensible para cuantificar mediante EAA-F y presenta el intervalo lineal más amplio respecto a los otros seis analitos. También se puede observar que el $\mathrm{Fe}$ y el Ni tienen sensibilidades parecidas.

Los valores de precisión y exactitud de las mediciones se presentan en el cuadro VI. La precisión se presenta como el porcentaje de la desviación estándar relativa (\% DER), el cual resultó ser, para los tres niveles de concentración de estudio y los siete analitos de trabajo $\leq 3 \%$, por lo que de acuerdo con Taverniers et al. (2004) los sistemas de medición de los siete elementos son precisos. Por otro lado, los porcentajes de exactitud calculados se encuentran comprendidos dentro de una diferencia del $\pm 15 \%$ con relación al valor real, criterio que indica como aceptable la exactitud del método desarrollado.

Respecto a los límites de detección y cuantificación determinados, los resultados se presentan en el cuadro VII. Para todos los casos se puede observar que el valor de LC de cada elemento coincide con el punto más bajo del intervalo lineal de concentraciones correspondiente, como lo sugieren Magnusson and Örnemark (2014). Estos resultados muestran que se puede cuantificar el contenido de $\mathrm{Ni}, \mathrm{Cu}, \mathrm{Zn}$ y Mn cuando sus concentraciones se encuentran en el orden de las decenas de $\mu \mathrm{g} / \mathrm{L}$, mientras que para el Fe, $\mathrm{Pb}$ y Cr la cuantificación por medio de EAA-F resultó ser menos sensible. En cuanto al límite de detección, el valor más bajo fue el obtenido para $\mathrm{Mn}$.

\section{Evaluación de interferencias}

Los resultados de la evaluación de interferencias de matriz de las muestras fortificadas, en los tres niveles de concentración y con los siete analitos de estudio, se resumen en el cuadro VIII, en el cual
CUADRO VI. EVALUACIÓN DE LA EXACTITUD Y DE LA PRECISIÓN DE LOS SIETE ANALITOS DE ESTUDIO $(n=5)$

\begin{tabular}{lcccr}
\hline \multirow{2}{*}{ Analito } & \multicolumn{2}{c}{ Precisión } & \multicolumn{2}{c}{ Exactitud } \\
\cline { 2 - 5 } & $\begin{array}{c}\text { Concentración } \\
(\mathrm{mg} / \mathrm{L})\end{array}$ & $\begin{array}{c}\text { DER } \\
(\%)\end{array}$ & $\begin{array}{c}\text { Concentración } \\
(\mathrm{mg} / \mathrm{L})\end{array}$ & $\begin{array}{c}\text { Exactitud } \\
(\%)\end{array}$ \\
\hline $\mathrm{Cr}$ & 0.5 & 3.0 & 0.7 & 98.4 \\
& 1.5 & 2.9 & 1.0 & 100.8 \\
& 2.5 & 1.3 & 3.5 & 97.1 \\
\hline $\mathrm{Pb}$ & 3.0 & 1.5 & 2.5 & 105.2 \\
& 7.0 & 0.4 & 5.0 & 100.3 \\
& 14.0 & 0.8 & 15.0 & 98.6 \\
\hline $\mathrm{Fe}$ & 0.5 & 0.7 & 0.2 & 100.2 \\
& 1.5 & 2.4 & 1.8 & 88.6 \\
& 3.5 & 0.3 & 2.5 & 83.4 \\
\hline $\mathrm{Ni}$ & 0.15 & 1.1 & 0.5 & 115.0 \\
& 1.0 & 1.4 & 1.5 & 108.7 \\
& 2.5 & 1.8 & 2.8 & 114.5 \\
\hline $\mathrm{Cu}$ & 0.5 & 2.3 & 0.3 & 97.7 \\
& 1.5 & 2.5 & 2.5 & 108.1 \\
& 3.8 & 1.3 & 3.5 & 104.1 \\
\hline $\mathrm{Zn}$ & 0.25 & 0.9 & 0.15 & 98.6 \\
& 0.45 & 1.0 & 0.40 & 103.7 \\
& 0.60 & 0.5 & 0.80 & 97.5 \\
\hline $\mathrm{Mn}$ & 0.08 & 2.2 & 0.5 & 103.0 \\
& 1.2 & 1.1 & 1.5 & 101.6 \\
& 2.0 & 0.6 & 2.5 & 106.0 \\
\hline
\end{tabular}

DER: desviación estándar relativa

puede observarse que las recuperaciones obtenidas se encuentran dentro del intervalo comprendido entre el 85 y $115 \%$, el cual es aceptado para considerar que se cuenta con una metodología libre de interferencias (Taverniers et al. 2004). Estos resultados demuestran que pueden hacerse buenas determinaciones de los 
CUADRO VII. VALORES DE LÍMITE DE DETECCIÓN (LD) Y LÍMITE DE CUANTIFICACIÓN (LC) DETERMINADOS POR MEDIO DE ESPECTROSCOPIA DE ABSORCIÓN ATÓMICACON FLAMA PARA CADAANALITO $(\mathrm{n}=7)$

\begin{tabular}{lccc}
\hline Analito & $\begin{array}{c}\text { Concentración utilizada } \\
(\mathrm{mg} / \mathrm{L})\end{array}$ & $\begin{array}{c}\mathrm{LD} \\
(\mathrm{mg} / \mathrm{L})\end{array}$ & $\begin{array}{c}\mathrm{LC} \\
(\mathrm{mg} / \mathrm{L})\end{array}$ \\
\hline $\mathrm{Cr}$ & 0.20 & 0.20 & 0.22 \\
$\mathrm{~Pb}$ & 0.20 & 0.10 & 0.23 \\
$\mathrm{Fe}$ & 0.04 & 0.12 & 0.20 \\
$\mathrm{Ni}$ & 0.05 & 0.05 & 0.06 \\
$\mathrm{Cu}$ & 0.02 & 0.05 & 0.11 \\
$\mathrm{Zn}$ & 0.05 & 0.05 & 0.08 \\
$\mathrm{Mn}$ & 0.02 & 0.03 & 0.05 \\
\hline
\end{tabular}

CUADRO VIII. EVALUACIÓN DE INTERFERENCIAS PARA CADAANALITO EN PRUEBAS DE FORTIFICACIÓN $(\mathrm{n}=3)$

\begin{tabular}{|c|c|c|}
\hline Elemento & $\begin{array}{c}\text { Concentración adicionada } \\
\text { (mg/L) }\end{array}$ & $\begin{array}{c}\text { Recuperación } \\
(\%)\end{array}$ \\
\hline \multirow[t]{3}{*}{$\mathrm{Cr}$} & 0.59 & 100.1 \\
\hline & 2.09 & 101.5 \\
\hline & 3.59 & 102.0 \\
\hline \multirow[t]{3}{*}{$\mathrm{Pb}$} & 1.15 & 103.9 \\
\hline & 7.15 & 102.3 \\
\hline & 15.15 & 95.8 \\
\hline \multirow[t]{3}{*}{$\mathrm{Fe}$} & 0.76 & 109.9 \\
\hline & 1.96 & 104.7 \\
\hline & 3.46 & 102.0 \\
\hline \multirow[t]{3}{*}{$\mathrm{Ni}$} & 0.13 & 106.4 \\
\hline & 1.83 & 97.5 \\
\hline & 3.23 & 94.2 \\
\hline \multirow[t]{3}{*}{$\mathrm{Cu}$} & 1.19 & 81.6 \\
\hline & 1.89 & 87.0 \\
\hline & 3.29 & 90.8 \\
\hline \multirow[t]{3}{*}{$\mathrm{Zn}$} & 0.18 & 94.7 \\
\hline & 0.28 & 81.1 \\
\hline & 0.56 & 78.9 \\
\hline \multirow[t]{3}{*}{$\mathrm{Mn}$} & 1.50 & 82.9 \\
\hline & 2.10 & 100.3 \\
\hline & 2.70 & 107.7 \\
\hline
\end{tabular}

siete elementos, incluso en presencia de una matriz compleja como una muestra geológica. Además, al estar presentes todos los elementos analizados (y algunos otros incluidos en la muestra geológica per se), se puede apreciar que éstos no causan interferencias significativas en los análisis de $\mathrm{Ni}, \mathrm{Cu}, \mathrm{Zn}$, $\mathrm{Mn}, \mathrm{Fe}, \mathrm{Pb}$ y $\mathrm{Cr}$.

\section{Intercomparación de metodologías}

Con el propósito de evaluar el efecto de la preparación de los materiales geológicos por medio de la digestión ácida asistida por microondas, así como la cuantificación de los siete elementos de interés mediante la técnica de EAA-F, se realizó la comparación de los resultados obtenidos de los análisis de EAA-F con los generados por la técnica de FRX-WD. Se decidió graficar en el eje de las $x$ la concentración del elemento determinada por FRX-WD y en el eje de las $y$ la concentración determinada por EAA-F (Fig. 1). Si bien los resultados obtenidos indican que para $\mathrm{Zn}, \mathrm{Cu}, \mathrm{Mn}, \mathrm{Fe}$ y $\mathrm{Pb}$ existe una respuesta lineal de la comparación de ambas técnicas, ya que los factores de correlación son $>0.99$, sólo para el $\mathrm{Zn}$, el Cu y el $\mathrm{Pb}$ se obtuvieron valores de pendiente que equivalen a porcentajes de reproducibilidad comprendidos entre el 85 y $110 \%$. Debido a esto, es posible afirmar que solamente estos tres elementos cumplen con los límites mínimo y máximo, establecidos para niveles de concentración del orden de las partes por millón en muestras reales, de acuerdo con los sugerido por la Comisión de Control Analítico y Ampliación de Cobertura (CCAyAC 2011).

Respecto al Mn y al Fe se obtuvieron valores de pendiente que no cumplen con esta condición, ya que las concentraciones de estos dos elementos determinadas por EAA-F fueron menores que las encontradas por FRX. Cabe mencionar que para llevar a cabo los análisis por EAA-F se requiere de muestras en estado líquido, por lo que en el presente trabajo fue necesario digerir previamente los materiales de trabajo. Debido a que los resultados obtenidos al fortificar los digeridos de los materiales geológicos no indicaron la presencia de interferencias para la cuantificación de los siete analitos de estudio, las bajas concentraciones de Mn y Fe determinadas por EAA-F se atribuyen a una deficiente digestión de los materiales geológicos. La incompleta solubilización de los materiales geológicos se atribuye a la presencia de altos contenidos de sílice ( $>34 \%$ ), en los que se encuentran asociados minerales de Fe y $\mathrm{Mn}$, por lo que, para solubilizarlos de manera completa es necesario utilizar un medio de digestión más fuerte, lo que implica el uso de HF.

Respecto al Ni y al Cr no fue posible la comparación en forma de gráfico de las dos metodologías de análisis; esto se debe a que las concentraciones presentes de estos dos elementos en los materiales geológicos no son lo suficientemente abundantes para ser cuantificadas por EAA-F, ya que durante la preparación de la muestra los analitos se diluyen. En el caso del Ni sólo fue posible realizar la comparación 

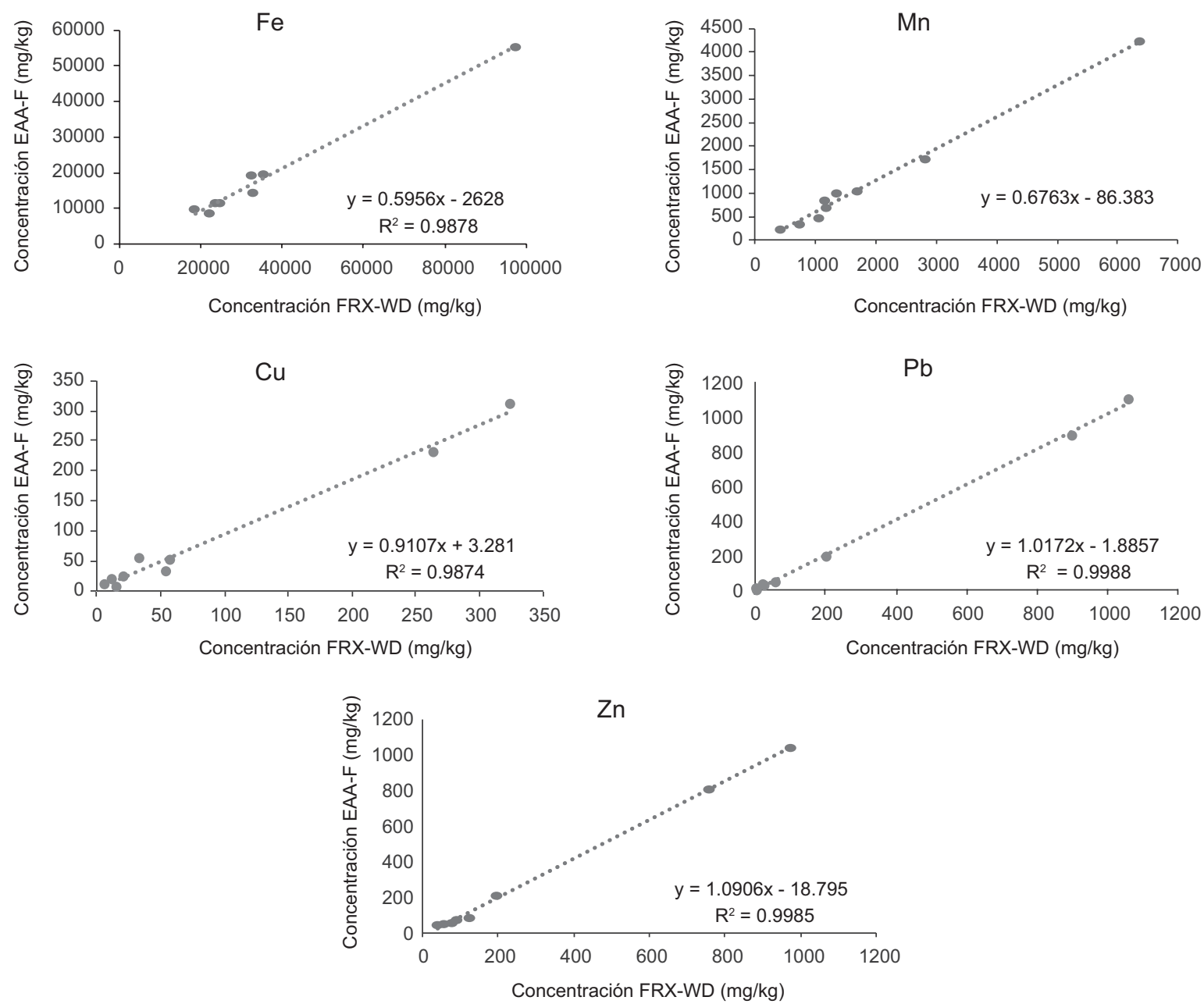

Fig. 1. Gráficos comparativos de los resultados obtenidos para $\mathrm{Fe}, \mathrm{Mn}, \mathrm{Cu}, \mathrm{Pb}$ y $\mathrm{Zn}$ por medio de fluorescencia de rayos $\mathrm{X}$ con dispersión de longitud de onda (FRW-WD) y espectroscopia de absorción atómica con flama (EAA-F) $(n=10)$

de las dos técnicas de análisis en el material llamado IGLgb3, en el cual los valores de concentración determinados por ambas técnicas fueron equivalentes: $10 \mathrm{mg} / \mathrm{kg}$ de Ni por medio de FRX y $10.5 \mathrm{mg} / \mathrm{kg}$ de Ni por EAA-F.

En las determinaciones de Fe y Mn en materiales geológicos por FRX existen pocas interferencias espectrales y las existentes por efecto de matriz son conocidas y corregibles. Los análisis se realizan sobre la muestra total sin la necesidad de llevar a cabo digestiones, lo que justifica los resultados obtenidos por FRX.

\section{Espectroscopia visible Optimización de las condiciones de trabajo}

De las tres concentraciones molares evaluadas de oFen para la formación del complejo con $\mathrm{Fe}^{2+}$, la de $2.5 \times 10^{-3} \mathrm{M}$ fue la que presentó la mayor sensibi- lidad (valor de pendiente más alto). Con relación al efecto del $\mathrm{pH}$ sobre la formación del complejo, los resultados obtenidos indicaron que no existe diferencia cuando se trabaja a $\mathrm{pH} 4.2$ y 9.0 , ya que las pendientes de las curvas de calibración del complejo $\left[\mathrm{Fe}(\mathrm{oFen})_{3}\right]^{2+}$ son iguales. En el caso particular del $\mathrm{Fe}$ total, no se encontró diferencia sobre la cuantificación de hierro al realizarla con hidroxilamina al 1 ó $2 \%$, ya que se obtuvieron valores de pendiente iguales. Sin embargo, se decidió trabajar con un $2 \%$ del reductor para realizar la optimización de la metodología y con ello garantizar la reducción completa del hierro en las muestras geológicas. Respecto a la determinación del contenido de $\mathrm{Fe}^{3+}$, de las tres concentraciones evaluadas de $\mathrm{SCN}^{-}$, la mejor respuesta analítica se obtuvo al trabajar con una concentración equivalente a $0.45 \mathrm{M}$. Cabe mencionar que con la determinación de $\mathrm{Fe}^{3+}$ a pH 4.0 no se obtuvo un comportamiento li- 
neal de la absorbancia en función de la concentración de hierro, mientras que al fijar el $\mathrm{pH}$ por debajo de 1.0 si se obtuvo dicho comportamiento requerido (cuadro IX).

\section{Determinación de los parámetros de desempeño}

Con las variables optimizadas de cada análisis (Cuadro III) se determinaron los intervalos lineales para cada analito, encontrándose que en todos los casos son de dos órdenes de magnitud, siendo las determinaciones de $\mathrm{Fe}$ total y de $\mathrm{Fe}^{2+}$ lineales hasta valores de concentración más bajos que los encontrados para $\mathrm{Fe}^{3+}$. Las tres determinaciones presentan sensibilidades semejantes, ya que los valores de pendiente de las tres curvas de calibración son muy parecidos entre sí (Cuadro X).

Los valores de precisión y exactitud de las tres determinaciones se presentan en el cuadro XI. En cuanto a la precisión se observa que, para las tres determinaciones y los tres niveles de concentración de estudio, todos los valores del porcentaje de la desviación estándar relativa fueron $\leq 3 \%$, por lo que se cumple con lo establecido por Taverniers et al. (2004) para considerar que el sistema de medición es preciso. Por otro lado, los valores de exactitud se encuentran muy cercanos al $100 \%$ sin exceder la diferencia del
CUADRO XI. EVALUACIÓN DE LA EXACTITUD Y DE LA PRECISIÓN DE LOS TRES ANALITOS $\operatorname{DE} \operatorname{ESTUDIO}(\mathrm{n}=5)$

\begin{tabular}{lccccc}
\hline \multirow{2}{*}{ Analito } & \multicolumn{2}{c}{ Precisión } & & \multicolumn{2}{c}{ Exactitud } \\
\cline { 2 - 3 } \cline { 6 - 6 } & $\begin{array}{c}\text { Concentración } \\
(\mathrm{mg} / \mathrm{L})\end{array}$ & $\begin{array}{c}\text { DER } \\
(\%)\end{array}$ & & $\begin{array}{c}\text { Concentración } \\
(\mathrm{mg} / \mathrm{L})\end{array}$ & $\begin{array}{c}\text { Exactitud } \\
(\%)\end{array}$ \\
\hline Fe total & 0.5 & 1.7 & 1.0 & 95.8 \\
& 7.0 & 1.6 & 5.0 & 94.6 \\
& 17.5 & 1.2 & 15.0 & 99.6 \\
\hline $\mathrm{Fe}^{2+}$ & 0.5 & 0.9 & 1.0 & 107.1 \\
& 7.0 & 2.9 & 5.0 & 102.5 \\
& 17.5 & 1.8 & 15.0 & 101.9 \\
\hline $\mathrm{Fe}^{3+}$ & 0.4 & 1.6 & 0.6 & 96.6 \\
& 7.0 & 1.3 & 9.0 & 100.2 \\
& 13.0 & 0.1 & 16.0 & 99.7 \\
\hline
\end{tabular}

DER: desviación estándar relativa

$\pm 15 \%$, por lo que es posible decir que se cuenta con metodologías exactas para la cuantificación de Fe total, $\mathrm{Fe}^{2+}$ y Fe ${ }^{3+}$.

Con relación a los límites de detección y cuantificación determinados, los resultados se presentan en el cuadro XII. Con base en los resultados obtenidos

CUADRO IX. EFECTO DE LAS CONCENTRACIONES DE LOS AGENTES DE COMPLEJACIÓN Y DEL pH SOBRE LA FORMACIÓN DE LOS COMPLEJOS DE HIERRO AL $95 \%$ DE CONFIANZA PARA 7 NIVELES DE CONCENTRACIÓN $(\mathrm{n}=3)$

\begin{tabular}{|c|c|c|c|c|}
\hline Analito (s) & Reactivo & Concentración & Pendiente (L/mg) & Efecto $\mathrm{pH}$ \\
\hline $\mathrm{Fe}^{2+}$ & oFen & $\begin{array}{l}4.4 \times 10^{-4} \mathrm{M} \\
8.0 \times 10^{-4} \mathrm{M} \\
2.5 \times 10^{-3} \mathrm{M}\end{array}$ & $\begin{array}{l}0.1677 \pm 0.0310 \\
0.2023 \pm 0.0311 \\
0.2316 \pm 0.0009\end{array}$ & $\begin{array}{l}\text { No se observó diferencia signifi- } \\
\text { cativa en los dos valores de } \mathrm{pH} \text { de } \\
\text { trabajo }\end{array}$ \\
\hline Fe total & $\mathrm{NH}_{2} \mathrm{OH}$ & $\begin{array}{l}1 \% \\
2 \%\end{array}$ & $\begin{array}{l}0.1984 \pm 0.0090 \\
0.1951 \pm 0.0109\end{array}$ & $\begin{array}{l}\text { No se observó diferencia signifi- } \\
\text { cativa en los dos valores de } \mathrm{pH} \text { de } \\
\text { trabajo }\end{array}$ \\
\hline $\mathrm{Fe}^{3+}$ & $\mathrm{SCN}^{-}$ & $\begin{array}{l}0.05 \mathrm{M} \\
0.20 \mathrm{M} \\
0.45 \mathrm{M}\end{array}$ & $\begin{array}{l}0.0162 \pm 0.0054 \\
0.0384 \pm 0.0113 \\
0.0635 \pm 0.0229\end{array}$ & $\begin{array}{l}\text { Sólo existe respuesta lineal cuando } \\
\text { el } \mathrm{pH} \text { del medio es }<1\end{array}$ \\
\hline
\end{tabular}

CUADRO X. ANÁLISIS DE REGRESIÓN LINEAL PARA LOS TRES ANALITOS AL $95 \%$ DE CONFIANZA PARA 10 NIVELES DE CONCENTRACIÓN $(n=3)$

\begin{tabular}{lcccc}
\hline Analito & $\begin{array}{c}\text { Intervalo lineal } \\
(\mathrm{mg} / \mathrm{L})\end{array}$ & $\begin{array}{c}\text { Ordenada } \\
\text { al origen }\end{array}$ & $\begin{array}{c}\text { Pendiente } \\
(\mathrm{L} / \mathrm{mg})\end{array}$ & $\begin{array}{c}\text { Coeficiente de } \\
\text { correlación }(\mathrm{r})\end{array}$ \\
\hline Fe total & $0.05-17.5$ & $-0.0007 \pm 0.0213$ & $0.1944 \pm 0.0025$ & 0.9997 \\
$\mathrm{Fe}^{2+}$ & $0.05-17.5$ & $0.0171 \pm 0.0209$ & $0.2043 \pm 0.0023$ & 0.9996 \\
$\mathrm{Fe}^{3+}$ & $0.15-17.5$ & $-0.0133 \pm 0.0165$ & $0.1846 \pm 0.0018$ & 0.9997 \\
\hline
\end{tabular}


CUADRO XII. VALORES DE LÍMITE DE DETECCIÓN (LD) Y LÍMITE DE CUANTIFICACIÓN (LC) DETERMINADOS POR MEDIO DE ESPECTROSCOPIA VISIBLE PARA CADA ANALITO $(n=7)$

\begin{tabular}{lccc}
\hline Analito & $\begin{array}{c}\text { Concentración } \\
\text { utilizada }(\mathrm{mg} / \mathrm{L})\end{array}$ & $\begin{array}{c}\mathrm{LD} \\
(\mathrm{mg} / \mathrm{L})\end{array}$ & $\begin{array}{c}\mathrm{LC} \\
(\mathrm{mg} / \mathrm{L})\end{array}$ \\
\hline Fe total & 0.01 & 0.02 & 0.05 \\
$\mathrm{Fe}^{2+}$ & 0.02 & 0.04 & 0.05 \\
$\mathrm{Fe}^{3+}$ & 0.05 & 0.12 & 0.14 \\
\hline
\end{tabular}

se observa que las especies de Fe presentan valores de $\mathrm{LD} \leq 0.12 \mathrm{mg} / \mathrm{L}$, mientras que los valores de LC se encuentran, de acuerdo con lo sugerido por Magnusson and Örnemark (2014), muy cercanos al valor de concentración más bajo de su respectivo intervalo lineal de concentraciones.

\section{Evaluación de interferencias}

Los resultados de la fortificación de la mezcla de los lixiviados de mina se muestran en el cuadro XIII, en el cual puede observarse que las recuperaciones se encuentran dentro del intervalo comprendido entre el 85 y $115 \%$, tal como lo establecen Taverniers et al. (2004) para considerar que se cuenta con una metodología libre de interferencias.

\section{CUADRO XIII. EVALUACIÓN DE INTERFERENCIAS PARA CADA ANALITO EN PRUEBAS DE FORTIFICACIÓN $(\mathrm{n}=3)$}

\begin{tabular}{lcc}
\hline Especie & $\begin{array}{c}\text { Concentración adicionada } \\
(\mathrm{mg} / \mathrm{L})\end{array}$ & $\begin{array}{c}\text { Recuperación } \\
(\%)\end{array}$ \\
\hline Fe total & 0.5 & 81.4 \\
& 50 & 90.4 \\
& 100 & 102.0 \\
\hline $\mathrm{Fe}^{2+}$ & 0.5 & 90.9 \\
& 50 & 95.4 \\
& 100 & 101.2 \\
\hline $\mathrm{Fe}^{3+}$ & 0.5 & 112.0 \\
& 50 & 107.0 \\
& 100 & 94.1 \\
\hline
\end{tabular}

\section{Intercomparación de metodologías}

Una forma de verificar si una metodología analítica es realmente confiable para aplicarse a un tipo de muestra definido, es la comparación directa de los resultados obtenidos con ella y otra metodología ya validada. En este caso, sólo se logró para la cuantificación de Fe total, ya que como técnica confirmatoria se utilizó ICP-OES, la cual de manera directa no hace especiación.

Al graficar los valores de concentración de Fe total determinados por medio de espectroscopia visible, en función de los respectivos valores de concentración del Fe determinado por ICP-OES de las 57 muestras de trabajo, se observó un comportamiento lineal (figura 2). El valor del coeficiente de determinación $\left(\mathrm{R}^{2}\right)$ es de 0.9952 , con un valor de pendiente muy cercano a la unidad. Debido a lo anterior se considera que los resultados generados por ambos sistemas de análisis son estadísticamente equivalentes, ya que no existe diferencia significativa (al $95 \%$ de confianza) entre las medias y los valores de desviación estándar de las concentraciones reportadas por ambos métodos. Con esto es posible decir que se cuenta con una metodología confiable y robusta para la cuantificación de Fe total en lixiviados de mina en el intervalo de concentraciones comprendidos entre 0.5 y $420 \mathrm{mg} / \mathrm{L}$ por medio de la técnica de espectroscopia visible.

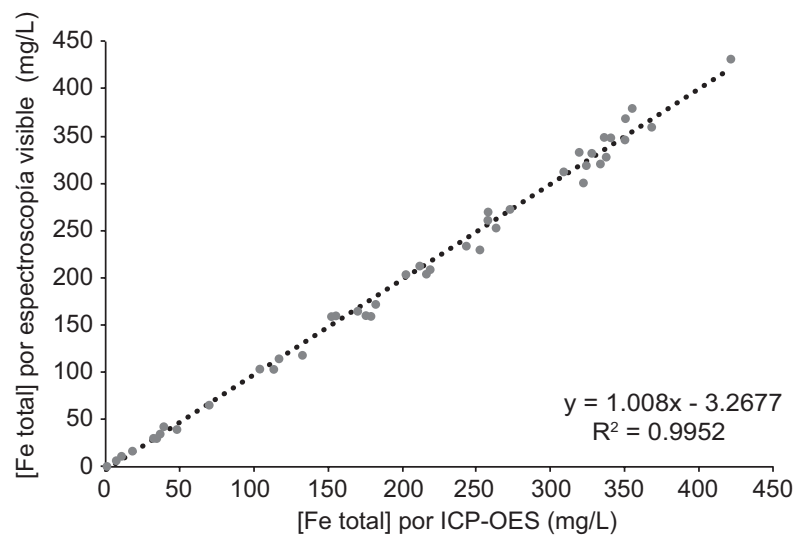

Fig. 2. Gráfico comparativo de los resultados obtenidos de Fe total por medio de espectroscopia de emisión atómica con plasma acoplado inductivamente (ICP-OES) y espectroscopia visible $(n=57)$

Sin embargo, ya que no se contó con otra técnica validada para determinar los contenidos de $\mathrm{Fe}^{2+}$ y $\mathrm{Fe}^{3+}$, se decidió evaluar al mismo tiempo el desempeño de las tres metodologías propuestas para hierro. Para ello se consideró: 1) cuantificar las concentraciones de Fe total y $\mathrm{Fe}^{2+}$ en las 57 muestras de los lixiviados con oFen; 2) calcular la diferencia de dichas concentraciones para obtener el contenido de $\mathrm{Fe}^{3+}$ esperado para cada muestra; 3) llevar a cabo la cuantificación de $\mathrm{Fe}^{3+}$ usando $\mathrm{SCN}^{-}$, y 4) realizar la comparación entre la concentración del 
$\mathrm{Fe}^{3+}$ esperado (calculado por diferencia) y el $\mathrm{Fe}^{3+}$ determinado con $\mathrm{SCN}^{-}$. En la figura 3 se presenta el gráfico obtenido cuando la concentración de $\mathrm{Fe}^{3+}$ esperada se grafica en función de la concentración de $\mathrm{Fe}^{3+}$ alcanzada con tiocianato. Como se puede observar, se presenta un comportamiento lineal en el intervalo de concentraciones comprendido entre 5 y $375 \mathrm{mg} / \mathrm{L}$, con un coeficiente de determinación de 0.9966 y con valor de pendiente prácticamente de la unidad. Esto indica que los resultados obtenidos por las metodologías son similares y confirma que no existen interferencias para la cuantificación de estas tres especies en las muestras de los lixiviados de mina utilizados.

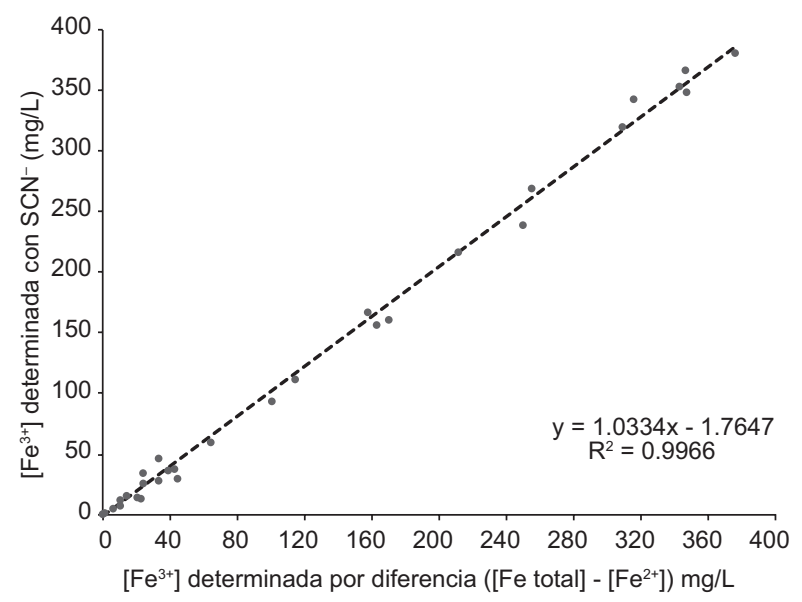

Fig. 3. Comparación de los contenidos de $\mathrm{Fe}^{3+}$ determinados por metodologías independientes en las muestras de lixiviados de mina $(\mathrm{n}=57)$

\section{CONCLUSIONES}

La optimización y adecuabilidad de las metodologías de EAA-F y espectroscopia visible descritas en el presente trabajo, permitieron conocer sus alcances para la determinación de algunas especies químicas en muestras de origen geológico de composición compleja tales como suelos, sedimentos, rocas y lixiviados de mina.

Mediante el uso de EAA-F se verificó que se cuenta con una metodología para analizar de manera secuencial, precisa, exacta y libre de interferencias los contenidos de $\mathrm{Fe}, \mathrm{Mn}, \mathrm{Cu}, \mathrm{Cr}, \mathrm{Ni}, \mathrm{Zn}$ y $\mathrm{Pb}$ en rocas, suelos y sedimentos en niveles de concentración del orden de partes por millón. La comparación de los resultados de las concentraciones obtenidas para los siete analitos de trabajo con EAA-F y FRX-WD, indicó que la metodología de digestión utilizada sólo funcionó eficientemente para los analitos $\mathrm{Cu}, \mathrm{Ni}, \mathrm{Zn}$ y $\mathrm{Pb}$ en los materiales geológicos en estudio.

Respecto a la espectroscopia visible, se lograron establecer las condiciones para determinar las concentraciones de $\mathrm{Fe}$ total, $\mathrm{Fe}^{2+}$ y $\mathrm{Fe}^{3+}$ presentes en lixiviados de mina, mediante reacciones de complejación. Se encontró que las tres metodologías son precisas y exactas, con intervalos dinámicos de concentración de tres órdenes de magnitud, con límites de detección del orden de partes por billón, y que, para este tipo de muestras, se encuentran libres de interferencias. La comparación de los resultados de las concentraciones obtenidas de Fe total por espectroscopia visible e ICP-OES muestra que hay reproducibilidad en los resultados para el intervalo de concentraciones de Fe comprendido entre 5 y $375 \mathrm{mg} / \mathrm{L}$.

\section{AGRADECIMIENTOS}

Los autores agradecen a Fabiola Vega García y a Ciro Eliseo Márquez Herrera por la realización de los análisis de ICP-OES. También se agradece a los revisores anónimos por sus atinados comentarios y sugerencias que ayudaron a enriquecer este trabajo.

\section{REFERENCIAS}

Arango A., Vélez C., Jaramillo Y., Valencia M. y Hernández A. (2012). Cuantificación de hierro ferroso en espinaca y harina fortificada: una aplicación para la industria de panificación. J. Eng. Technol. 1 (1), 18-25.

Ata S., Hamid F., Ahmed M., Hamid M., Ahmed S. y Wadood A. (2015). A method optimization study for atomic absorption spectrophotometric determination of total zinc in insulin using direct aspiration technique. Alexandria J. Med. 51 (1), 19-23.

DOI: 10.1016/j.ajme.2014.03.004

Balasubramanian S. y Pugalenthi V. (1999). Determination of total chromium in tannery wastewater by inductively coupled plasma-atomic emission spectrometry, flame atomic absorption spectrometry and UV-visible spectrophotometric methods. Talanta 50 (3), 457-467. DOI: 10.1016/S0039-9140(99)00135-6

Brouwer P. (2013). Teoría de FRX. 3a ed. PANalytical, Almelo, Holanda, 65 pp.

CCAyAC (2011). CCAyAC-P-058. Criterios para la validación de métodos fisicoquímicos. Guía técnica. Comisión de Control Analítico y Ampliación de Cobertura, Ciudad de México, México, 20 pp. 
CENAM-EMA (2008). Guía técnica sobre trazabilidad e incertidumbre en las mediciones analíticas que emplean la técnica de espectrofotometría de ultravioleta-visible. Guía técnica. Centro Nacional de Metrología-Entidad Mexicana de Acreditación, Querétaro, México, 69 pp.

Charlot G. (1964). Colorimetric determination of element (principles and methods). Elsevier, Nueva York, EUA, 449 pp.

Chen J. y Teo C. (2001). Determination of cadmium, copper, lead and zinc in water samples by flame atomic absorption spectrometry after cloud point extraction. Anal. Chim. Acta. 450, 215-222.

DOI: $10.1016 / \mathrm{S} 0003-2670(01) 01367-8$

Christian G.D. (2009). Química analítica. McGraw Hill, Ciudad de México, México, 828 pp.

Cravotta C.A. y Trahan M.K. (1999). Limestone drains to increase $\mathrm{pH}$ and remove dissolved metals from acidic mine drainage. Appl. Geochem. 14, 581-606. DOI: 10.1016/S0883-2927(98)00066-3

Gao H., Bai J., Xiao R., Liu P., Jiang W. y Wang J. (2013). Levels, sources and risk assessment of trace elements in wetland soils of a typical shallow freshwater lake, China. Stoch. Env. Res. Risk A. 27, 275-284. DOI: $10.1007 / \mathrm{s} 00477-012-0587-8$

Gómez-Nieto B., Gismera M., Sevilla M. y Procopio J. (2015). Fast sequential multi-element determination of major and minor elements in environmental samples and drinking waters by high-resolution continuum source flame atomic absorption spectrometry. Anal. Chim. Acta. 854, 13-19.

DOI: 10.1016/j.aca.2014.10.051

Gray N.F. (1997). Environmental impact and remediation of acid mine drainage: a management problem. Environ. Geol. 30 (1-2), 62-71.

DOI: $10.1007 / \mathrm{s} 002540050133$

Harris D.C. (2013). Análisis químico cuantitativo. 3a ed. Reverté, Barcelona, España, 774 pp.

Harvey A.E., Smart J.A. y Amis E.S. (1955). Simultaneous spectrophotometric determination of Iron (II) and total Iron with 1,10-phenanthroline. Anal. Chem. 27, 26-29. DOI: 10.1021/ac60097a009

Jennings S.R., Dollhopf D.J. e Inskeep W.P. (2000). Acid production from sulfide minerals using hydrogen peroxide weathering. Appl. Geochem. 15, 247-255. DOI: 10.1016/S0883-2927(99)00041-4

Jimeno T. (2017). Metodología de análisis químico de escorias del proceso de obtención de oro y plata Merrill-Crowe utilizando espectroscopia de emisión atómica con plasma acoplado inductivamente (ICPOES). Tesis de Licenciatura. Facultad de Química, Universidad Nacional Autónoma de México, Ciudad de México, México, 91 pp.
Lozano R. y Bernal J.P. (2005). Characterization of a new set of eight geochemical reference materials for XRF, major and trace element analysis. Rev. Mex. Cienc. Geo. 22 (3), 329-344.

Magnusson B. and Örnemark U., eds. (2014). Eurachem guide: The fitness for purpose of analytical methods - A laboratory guide to method validation and related topics. 2a ed. Eurachem, 62 pp. [en línea]. https://www.eurachem.org/images/stories/Guides/pdf/ MV_guide_2nd_ed_EN.pdf 02/05/2018

Miller J.N. y Miller J.C. (2002). Estadística y quimiometría para química analítica. 4a ed. Prentice Hall, Madrid, España, 278 pp.

Potts P.J., Tindle A.G. y Webb P.C. (1992). Geochemical reference material compositions. CRC Press, Boca Raton, Florida, EUA, 313 pp.

Sastre J., Vidal S. y Rauret G. (2002). Determination of Cd, $\mathrm{Cu}, \mathrm{Pb}$ and $\mathrm{Zn}$ in environmental samples: Microwave assisted total digestion versus aqua regia and nitric acid extraction. Anal. Chim. Acta. 462, 59-72.

DOI: $10.1016 / \mathrm{S} 0003-2670(02) 00307-0$

Singh V. y Agrawal H.M. (2012). Qualitative soil mineral analysis by EDXRF, XRD and AAS probes. Radiat. Phys. Chem. 81, 1796-1803.

DOI: 10.1016/j.radphyschem.2012.07.002

Skoog D.A., Holler M.J. y Nieman T.A (2001). Principios de análisis instrumental. 5a ed. McGraw-Hill, Madrid, España, 1024 pp.

Soodan R.K., Pakade Y.B., Nagpal A. y Katnoria J.K. (2014). Analytical techniques for estimation of heavy metals in soil ecosystem: A tabulated review. Talanta 125, 405-410. DOI: 10.1016/j.talanta.2014.02.033

Soylak M., Tuzen M., Santos A., Andrade M.G. y Costa S.L. (2007). Optimization of microwave assisted digestion procedure for the determination of zinc, copper and nickel in tea samples employing flame atomic absorption spectrometry. J. Hazard. Mater. 149, 264268. DOI: $10.1016 /$ j.jhazmat.2007.03.072

Taverniers I., de Loose M. y van Bockstaele E. (2004). Trends in quality in the analytical laboratory. II. Analytical method validation and quality assurance. TrAC-Trend. Anal. Chem. 23, 535-552.

DOI: $10.1016 /$ j.trac.2004.04.001

USEPA (1996a). Test Method 3050B. Acid digestion of sediments, sludges, and soils. United States Environmental Protection Agency [en línea]. https://www. epa.gov/sites/production/files/2015-06/documents/ epa-3050b.pdf 02/05/2018

USEPA (1996b). Test Method 3052. Microwave assisted acid digestion of siliceous and organically based matrices. United States Environmental Protection Agency [en línea]. https://www.epa.gov/sites/production/ files/2015-12/documents/3052.pdf 02/05/2018 
Walpole R.E., Myers R.H., Myers S.L. y Ye K. (2012). Probabilidad y estadística para ingeniería y ciencias. 9a ed. Pearson Educación, Ciudad de México, México, $816 \mathrm{pp}$.

Walton H.F. y Reyes J. (1983). Análisis químico e instrumental moderno. Reverté, Barcelona, España, 410 pp.

Wood J.T. y Mellon M.G. (1941). Thiocyanate method for Iron. A spectrophotometric study. Ind. Eng. Chem. Anal. Ed. 13 (8), 551-554.

DOI: $10.1021 / 1560096 \mathrm{a} 013$
Zamora O. (2003). Evaluación del riesgo asociado a jales mineros. Comportamiento de metales pesados y arsénico. Tesis de Maestría. Facultad de Química, Universidad Nacional Autónoma de México, Ciudad de México, México, 152 pp. 